CERN-EP/99-147

11 October 1999

\title{
MICRO-PATTERN GAS DETECTORS
}

Fabio Sauli

CERN, CH-1211 Geneva, Switzerland

\begin{abstract}
Micro-strip gas chambers, with their excellent localization properties, high rate capability and good granularity, have been adopted by many experiments in particle physics. Two recurrent problems however have been reported: a slow degradation under sustained irradiation (or aging), and the rare but devastating occurrence of discharges. New breeds of detectors aim at improving on these crucial points; the micro-dot, CAT, micromegas, the gas electron multiplier are examples. Very performing, they are moreover robust and reliable. Two-stage devices, making use of a gas electron multiplier as first element, permit larger gains in presence of high rates and heavily ionizing tracks. Possible promising future developments in the field are outlined.
\end{abstract}

Invited review talk at the

5th Conference on Position Sensitive Detectors

University College London, September 13-17, 1999

Corresponding author: fabio.sauli@cern.ch 


\section{THE CHALLENGE}

The development of large area position-sensitive detectors, capable of withstanding radiation fluxes approaching a MHz mm $\mathrm{mm}^{-2}$, is motivated in particle physics by the quest of rare events, embedded in a huge, uninteresting background. In medical diagnostics, similar requirements arise from the efforts to improve resolution. This has resulted in recent times in the introduction of innovative devices, as well as in systematic studies aimed at understanding and improving the radiation hardness of existing detectors.

\section{EXPECTATIONS AND DECEPTIONS}

After the success of devices based on the multiwire proportional chamber, the micro-strip gas chambers (MSGC), introduced in 1988 [1] appeared as a very promising innovation. Consisting of a set of thin metal strips laid on an insulating substrate, alternatively connected as anodes and cathodes, the devices can reach large proportional gains with excellent energy resolution. The photo-lithographic technology used for manufacture permits a granularity at least an order of magnitude better than wire systems, with the corresponding improvement in rate and multi-track capability. A decade of intense efforts to optimize geometry, substrates and operating gases, confirmed the expected major performances of the detector: a localization accuracy better than $40 \mu \mathrm{m}$ rms, and a rate capability exceeding $10^{5} \mathrm{~Hz} \mathrm{~mm}^{-2}$ [2]. This has motivated the adoption of large arrays of MSGCs as tracking device in physics experiments, as for example HERMES [3] and HERA-B [4] at DESY, and CMS at CERN [5].

Systematic laboratory search, and some traumatic beam exposures, revealed however two fundamental sources of degradation of the MSGC performances. The first is the creation of polymers in the gas under effect of sustained avalanches, with the formation of insulating layers on electrodes, affecting the gain. The process was expected to occur, from previous experience with wire chambers, but not with the swiftness and the degree of sensitivity to residual organic pollutants experimentally found [6]. A laborious search for "clean" components and gases resulted in sets of recommendations as to the materials that could be used for obtaining an acceptable lifetime in MSGC exposed to high radiation fluxes [7]. It remains however to be verified if these clean conditions, met in the laboratory, can be maintained in complex systems.

The second problem encountered is more crucial: the rare occurrence of breakdowns, often fatal for the fragile structures. The discharge probability is voltage dependent, and largely enhanced by the exposure to high fluxes and heavily ionizing particles [8]. In realistic beam operating conditions, the presence of ionizing background (showers, neutrons, nuclear fragments) can result in the fast degradation of plates, an observation that led the HERA-B experiment to abandon the original MSGC option [9].

\section{INVESTIGATING THE PROBLEMS}

Several authors, mainly using soft X-ray sources or generators, have studied the dependence of maximum gain on rate in gaseous detectors. A compilation of results obtained with two models of micro-strip, a micro-gap (MGC) and two parallel plate counters (PPAC and micromegas) is shown in 
Fig. $1[10,11]$. Aside from a different initial value at low rates, all devices have as common behavior a fast decrease of maximum gain on exceeding $10^{3}-10^{4} \mathrm{~Hz}$ $\mathrm{mm}^{-2}$. This has led to conjecture the existence of a universal boundary in the gain-rate plane, somewhat improperly named Raether limit, delimiting a forbidden zone (dashed area in the figure [12]). The problem with the classic explanation describing the transition from proportional avalanche to streamer, followed by a discharge, is that it relies on exceeding a threshold value for the total charge $\left(\sim 10^{8}\right)$. In most micro-pattern devices, the clearing time of electrons and ions, a few $\mu$ s at most, is too fast to allow rate-dependent charge accumulations. The authors of Ref. [12] speculate on the existence of insulating clusters on cathodes (dirt or oxides), charging up and inducing field emission. It is however rather puzzling that the discharge boundary can depend so little on physical parameters, such as the shape and nature of the electrodes, in a wide range of devices. A better understanding of this point seems mandatory.

A second, and in many cases more serious problem is the breakdown induced by highly ionizing tracks; the limiting gain appears to be well below the value set by high rates, and is therefore the dominant factor. Fig. 2 shows the typical behavior of a MSGC. Gain and efficiency for minimum ionizing tracks are provided as a function of cathode voltage, as well as the discharge probability induced by highly ionizing tracks (6 MeV alpha particles internally emitted by ${ }^{220} \mathrm{Rn}$ [13]). The discharge probability increases rapidly above gains around 2000, marginal for efficient operation. Given the average number of charges released by the source, few tens of thousand, one reaches indeed the known value of the Raether limit. Whilst not all experiments have a highly ionizing tracks background, this is a common occurrence in high-energy physics.

\section{PROMISING ALTERNATIVES}

Innovative detector designs have been developed recently, with encouraging performances and higher reliability. The micro-dot chamber is a true pixel device, with impressive rate capability and very high gains [14, 15]. Manufactured however using silicon technology, it remains rather expensive and limited in size. The micromegas [16], a thin-gap parallel plate counter, shown schematically in Fig. 3, consists in a thin metal mesh, stretched above a readout electrode, at a distance of 50 to $100 \mu \mathrm{m}$. Regularly spaced supports (insulating fibers or pillars) guarantee the uniformity of the gap. A high field is applied across the multiplying gap, and electrons released in the upper drift region are collected and multiplied. Operation at very high particle fluxes has been demonstrated [11, 17]. Fig. 4 gives an example of measured detector current at increasing X-ray fluxes; the curves are parallel, demonstrating good proportionality over a wide dynamic range. The discharge boundary, already discussed in the previous section, is also seen.

The "Compteur à Trous" or CAT $[18,19]$ consists basically in a single hole drilled through a metallic foil, with an anode at the bottom (Fig. 5). Proportional gains up to $10^{4}$ with surprisingly good energy resolution have been demonstrated. Several variations of the CAT structure have been developed, with multiple holes and insulating plate between anode and cathode in order to improve the mechanical stability and easy the construction. 
One of them, named micro-CAT, is successfully used for medical and industrial imaging applications [20].

The gas electron multiplier (GEM, Fig. 6) is a thin insulating foil, metalclad on both sides and perforated by a regular dense matrix of holes [21]. Upon application of a difference of potential, a high dipole field develops in the holes; electrons released by ionization in the gas drift in the high field through the open channels, multiply and transfer to a charge collecting electrode. GEMs operate in a large choice of gases, including non-flammable mixtures of argoncarbon dioxide, with gains exceeding $10^{4}$. High rate capability, good efficiency and position accuracy for detection of minimum ionizing particles have been demonstrated $[22,23]$. As the previous devices, on exposure to alpha particles, GEM suffers from a limitation in gain. In a systematic investigation, the authors of Ref. [13] have demonstrated indeed that all single-stage micro-pattern detectors, with the possible exception of the micro-dot, behave similarly, with a discharge probability increasing quickly above gains of a few thousand. In this respect, they are no better than MSGCs, even though they are far less fragile and easier to manufacture.

The discharge can be prevented to some extent coating the sharp edges of the cathodes with a thin insulator [24], an observation contradicted by other work [13]. A more effective solution is to remove the cathodes from the gas altogether, as in the so-called asymmetric [25] or virtual cathode chamber [26]. Problems of availability of the thin, controlled resistivity substrates required by this device have so far hindered the diffusion of the technology.

Because of the larger area of electrodes, the new micro-pattern detectors are far less sensitive to aging than micro-strips. Lifetimes equivalent to several years of use in harsh conditions have been verified for micromegas [11] and GEM [27], even making use of rather conventional fiberglass and epoxy assembly methods.

\section{TWO IS BETTER THAN ONE}

A way to obtain larger gains in gaseous structures, cascading several multipliers, was found time ago with the multi-step chamber [28]. More recently, the authors of Ref. [29] have made similar observations combining a parallel plate multiplier with a micro-dot. Operating at low pressures, gains above $10^{6}$ could be reached, sufficient for single photo-electron detection.

Multiple structures can be easily implemented using GEMs. Mounted in front of a MSGC, the device permits higher overall gains; alternatively, given the required gain, the voltage on both structures can be considerably reduced, largely improving reliability [30]. This solution has been adopted to rescue the tracker in the HERA-B experiment; several hundred large size GEM foils have been manufactured at CERN and added to the existing MSGC plates [9].

The double-GEM, Fig. 7, achieves similar performances without the use of the fragile MSGC. Systematic studies of amplification as a function of fields and geometry show that gains above $10^{5}$ can be reached, with only a small loss at extreme rates, see Fig. 8 [31]. A printed circuit board, collecting the electrons released from the second multiplier, is used for detection. Exposed to heavily ionizing particles, a double GEM detector can be operated, before the onset of discharges, at gains above $10^{4}$, an order of magnitude higher that single devices [13]. Two-dimensional localization can be achieved with a double-level circuit, 
manufactured using the same technology used for the GEMs, as read-out element [32]. The unique feature of this approach is that both readout electrodes are operated at ground potential. The picture in Fig. 9 shows an example of Xray absorption micro-radiography recorded with the detector; the position accuracy is better than $100 \mu \mathrm{m}$ [33].

The difference between optical and electrical transparency, combined with the observation of high gain in pure noble gases [34], suggested the use of several GEMs in cascade for the detection of electrons produced on solid photocathodes [35]. Fig. 10 provides gain curves obtained with a triple GEM in several gas mixtures that could be used in conjunction with bialkali photocathodes [36]. The use of a reverse geometry, with the photosensitive material deposited directly on the top of the GEM electrode thus completely eliminating photon feedback problems, is also being investigated [37]. Another interesting prospect, presently under study in several groups, is to deploy a set of GEM modules as read-out elements for time projection chambers. Possible advantages in this case include a large intrinsic ion feedback suppression, an improved two-track resolution and a higher rate capability.

\section{GUESSING THE FUTURE}

The low density of gaseous media sets basic limitations to the performances of detectors. Statistical fluctuations in the energy loss result in a wide, asymmetric spectra, and, in the thin layers required for fast response, in poor efficiency and position accuracy, quickly degrading with the incidence angle. Operation at pressures higher than atmospheric is possible, but implies the use of containment vessels adding unacceptable amount of material to the experiment. A very interesting possibility is to exploit secondary electron emission from cathodes, a process well known in vacuum, hindered however by back-scattering in presence of gas molecules. Good secondary emitters are low density layers of $\mathrm{KCl}, \mathrm{KBr}, \mathrm{LiF}$, CsI [38]; for a review of secondary emission in gaseous detectors see for example Ref. [39].

In a gas counter having the cathode coated with a columnar CsI layer, around $200 \mu \mathrm{m}$ thick, the authors of ref. [40] have demonstrated a substantial enhancement of the detected charge signal. In a more tantalizing device, realized with wires embedded in a thick low-density emitter and operating in vacuum, large secondary emission followed by multiplication has been observed [38]. Despite the marginal efficiency obtained so far, exploiting the secondary emission process with its intrinsic independence on the incidence angle and sub-nanosecond timing remains a very challenging possibility for future detection systems. 


\section{REFERENCES}

[1] A. Oed, Nucl. Instrum. and Meth. A263 (1988) 351.

[2] F. Sauli, Nucl. Phys. 61B (1998) 236.

[3] J.J. van Hunen, Nucl. Instrum. and Meth. A 409 (1998) 95.

[4] T. Zeuner, Nucl. Instrum. and Meth. A 392 (1997) 105.

[5] CMS Technical Design Report-The tracker project, CERN/LHCC 98-6 (1998).

[6] R. Bouclier et al., Nucl. Instrum. and Meth. A350 (1994) 464.

[7] R. Bouclier et al., Nucl. Instrum. and Meth. A381 (1996) 289.

[8] R. Bouclier et al., Nucl. Instrum. and Meth. A365 (1995) 65.

[9] B. Schmidt, Nucl. Instrum. and Meth. A 419 (1998) 230.

[10] P. Fonte et al., Nucl. Instrum. and Meth. A 419 (1998) 405.

[11] G. Puill et al., IEEE Trans. Nucl. Sci. NS-46 (1999) 594.

[12] P. Fonte et al., IEEE Trans. Nucl. Sci. NS-46 (1999) 91.

[13] A. Bressan et al., Nucl. Instrum. and Meth. A424 (1998) 321.

[14] S.F. Biagi et al., Nucl. Instrum. and Meth. A371 (1995) 12.

[15] S. Biagi et al., Nucl. Instrum. and Meth. A 419 (1998) 438.

[16] I. Giomataris et al., Nucl. Instrum. and Meth. A376 (1996) 29.

[17] Y. Giomataris, Nucl. Instrum. and Meth. A 419 (1998) 239.

[18] F. Bartol et al., J. Phys. III France 6 (1996) 337.

[19] G. Chaplier et al., Nucl. Instr. and Meth. A426 (1999) 339.

[20] A. Sarvestrani et al., Nucl. Instrum. and Meth. A 419 (1998) 444.

[21] F. Sauli, Nucl. Instrum. and Meth. A386 (1997) 531.

[22] J. Benlloch et al., Nucl. Instrum. and Meth. A 419 (1998) 410.

[23] A. Bressan et al., Nucl. Instrum. and Meth. A425 (1999) 262.

[24] R. Bellazzini et al., Nucl. Instrum. and Meth. A398 (1998) 426.

[25] G. Cicognani et al., IEEE Trans. Nucl. Sci. NS-45 (1998) 249.

[26] M. Capeáns et al., Nucl. Phys. B 61B (1998) 17.

[27] S. Bachmann et al., Proc. Micro-Pattern Gas Detectors Workshop (Orsay 28-30 June 1999).

[28] G. Charpak et al., Phys. Letters 78 B (1978) 523.

[29] A. Breskin et al., Nucl. Instrum. and Meth. A394 (1997) 21.

[30] R. Bouclier et al., Nucl. Instrum. and Meth. A396 (1997) 50.

[31] S. Bachmann et al., CERN-EP/99-48. Subm. Nucl. Instr. and Meth. (1999).

[32] A. Bressan et al., Nucl. Instrum. and Meth. A425 (1999) 254.

[33] A. Bressan et al., IEEE Trans. Nucl. Sci. NS-46 (1999) 86.

[34] A. Bressan et al., Nucl. Instrum. and Meth. A423 (1998) 119.

[35] G. Garty et al., Nucl. Instrum. and Meth. A433 (1999) 476.

[36] A. Breskin et al., Proc. Micro-Pattern Gas Detectors Workshop (Orsay 2830 June 1999) .

[37] E. Shefer et al., Nucl. Instr. and Meth. A419 (1998) 612.

[38] M.P. Lorikian et al., Nucl. Instr. and Meth. A340(19940625.

[39] A. Breskin, Nucl. Physics B44(1995)351.

[40] H.S. Cho et al., IEEE Trans. Nucl. Sci. NS-45 (1998) 275. 
Fig. 1: Maximum gain vs. rate for several micro-pattern detectors. A dashed line delimits the forbidden region.

Fig. 2: Gain, efficiency for minimum ionizing particles and discharge rate on internal alpha particles as a function of voltage in a MSGC. 


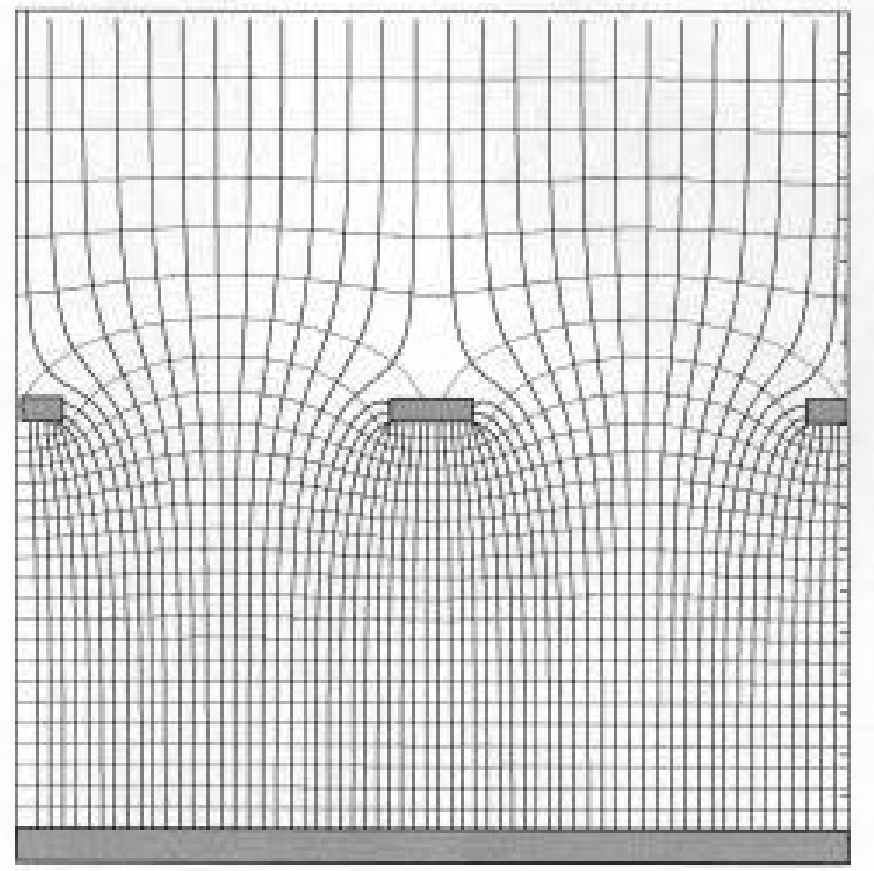

Fig. 3: Electric field structure in micromegas. The multiplying gap is typically 50 to $100 \mu \mathrm{m}$ thick.

Fig. 4: Detected current in micromegas as a function of voltage, for increasing radiation fluxes (6 $\mathrm{keV} \mathrm{X}$-rays). 
Fig. 5: Schematics and field structure in the CAT detector.

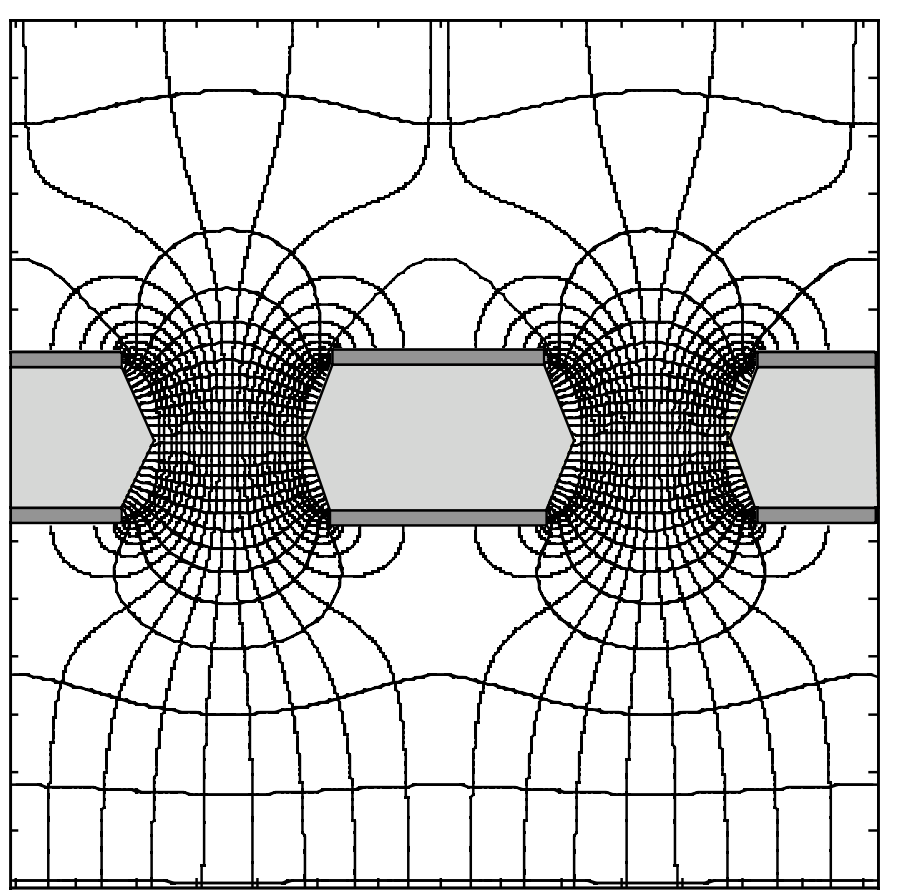

Fig. 6: Basic structure and electric field in the GEM. 


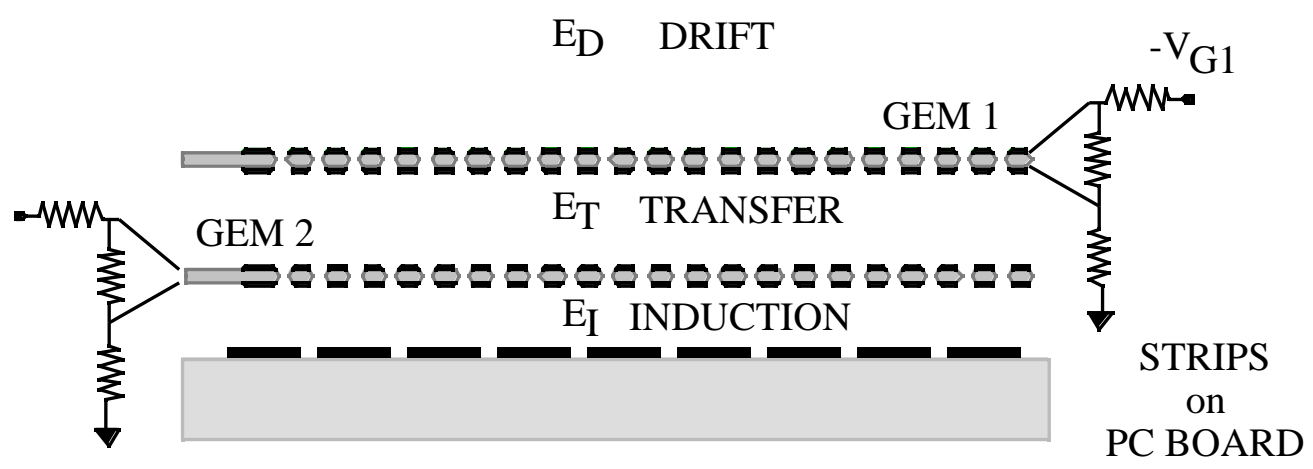

Fig. 7: Schematics of the double GEM detector with PCB readout.

Fig. 8: Current as a function of voltage in the double GEM detector, at increasing $\mathrm{X}$-ray fluxes. 


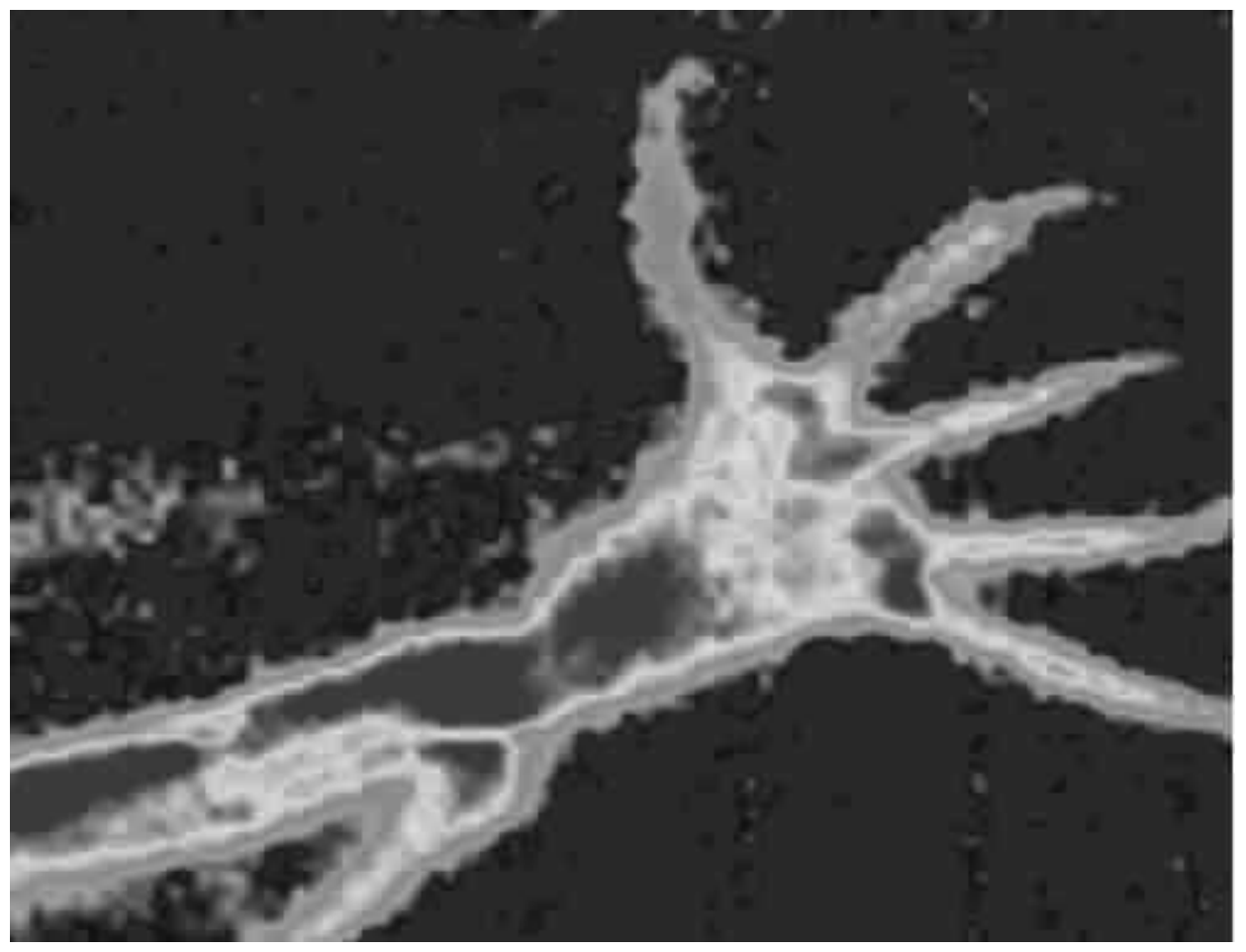

Fig. 9: X-ray absorption radiography of a micro-mammal foot, recorded with the bi-dimensional double GEM detector.

Fig. 10: Gain as a function of total voltage in the triple-GEM detector, for various photocathode-safe gas mixtures. 